\title{
Hybridized Plasmons in 2D Nano-slits: From Graphene to Anisotropic 2D Materials
}

\author{
P. A. D. Gonçalves, ${ }^{* \dagger, t, \uparrow}$ Sanshui Xiao, ${ }^{\dagger, \uparrow}$ N. M. R. Peres, ${ }^{\S}$ and \\ N. Asger Mortensen*,,$+ \|, \mathbb{\emptyset}$ \\ $\dagger$ Department of Photonics Engineering, Technical University of Denmark, DK-2800 \\ Kgs. Lyngby, Denmark \\ $\ddagger$ Center for Nano Optics, University of Southern Denmark, Campusvej 55, DK-5230 \\ Odense M, Denmark \\ \Center for Nanostructured Graphene, Technical University of Denmark, DK-2800 \\ Kgs. Lyngby, Denmark \\ $\S$ Department of Physics and Center of Physics, and QuantaLab, University of Minho, \\ PT-4710-057, Braga, Portugal \\ || Danish Institute for Advanced Study, University of Southern Denmark, Campusvej 55, \\ DK-5230 Odense M, Denmark \\ E-mail: padgo@fotonik.dtu.dk; asger@mailaps.org
}

\begin{abstract}
Plasmon coupling and hybridization in complex nanostructures constitutes a fertile playground for controlling light at the nanoscale. Here, we present a semi-analytical model to describe the emergence of hybrid plasmon modes guided along 2D nano-slits. In particular, we find two new coupled plasmonic resonances arising from symmetric and antisymmetric hybridizations of the edge plasmons of the constituent half-sheets. These give rise to an antibonding and a bonding mode, lying above and below the
\end{abstract}


energy of the bare edge plasmon. Our treatment is notably generic, being able to account for slits of arbitrary width, and remains valid irrespective of the $2 \mathrm{D}$ conductive material (e.g., doped graphene, 2D transition metal dichalcogenides, or phosphorene). We derive the dispersion relation of the hybrid modes of a $2 \mathrm{D}$ nano-slit along with the corresponding induced potential and electric field distributions. We also discuss the plasmonic spectrum of a $2 \mathrm{D}$ slit together with the one from its complementarity structure, that is, a ribbon. Finally, the case of a nano-slit made from an anisotropic 2D material is considered. Focusing on black phosphorus (which is highly anisotropic), we investigate the features of its plasmonic spectrum along the two main crystal axes. Our results offer insights into the interaction of plasmons in complex 2D nanostructures, thereby expanding the current toolkit of plasmonic resonances in $2 \mathrm{D}$ materials, and paving the way for the emergence of future compact devices based on atomically thin plasmonics.

\section{Keywords}

two-dimensional materials, plasmonics, graphene plasmons, hybridization, black phosphorus, nanostructures

Surface plasmons, collective oscillations of the electronic density in conductors, have been under the spotlight of the nanophotonics community over the last $\operatorname{decade}^{1}$ owing to their ability to confine optical fields below the diffraction limit. ${ }^{213}$ As of today, the annual number of plasmon-related publications is approaching a five-digit figure. ${ }^{4}$ Moreover, with the emergence of new opportunities on the horizon - such as plasmons in layered two-dimensional (2D) materials ${ }^{5 / 7}$ and quantum plasmonics ${ }^{8 \mid 9}$ the interest in the subject is likely to remain elevated over the next decade. ${ }^{4}$

The potential surface plasmons have to squeeze light into subwavevelength regimes leads to large electric field enhancements, which can be of several orders of magnitude, thereby 
promoting strong light-matter interactions in nanoscale environments. On the other hand, the local increase in the electric field strength near plasmonic nanostructures makes them particularly well-suited for biochemical sensing and surface-enhanced Raman scattering (SERS) applications. 10 13 Other ensuing phenomena includes the manifestation of large nanooptical forces, $\frac{14 \mid 15}{15}$ enhanced nonlinearities, $\frac{16 \mid 17}{1}$ and modification of the spontaneous emission rate of quantum emitters. $\frac{8|18| 19}{18}$

It is well known that the degree of field localization attained by surface plasmons is substantially higher within small gaps between metallic structures, e.g., inside metal grooves, 20121 plasmonic dimers and bow-ties, 2223 or in the so-called particle on a mirror geometry..$^{24 \mid 25}$ Indeed, in the latter configuration, sub-nanometric gaps with extremely small modal volumes have been reported using either self-assembled molecular monolayers ${ }^{26 / 27}$ or atomically thin 2D crystals. $\frac{28 \mid 29}{20 w e v e r, ~ a n d ~ d e s p i t e ~ i t s ~ m a n y ~ a l l u r i n g ~ p r o p e r t i e s, ~ t h r e e-d i m e n s i o n a l ~(3 D) ~}$ metal-based plasmonics currently faces two main obstacles which have been hampering its natural evolution from research laboratories to everyday technological devices relying on surface plasmons. The first, and often the most important, is unarguably the relatively short life-time of plasmons, since metals are inherently lossy. The other is associated with the limited tunability of the plasmon resonance, that tends to remain essentially fixed for a given geometry and material. In this panorama, graphene and other 2D materials beyond graphene (for instance, doped transition metal dichalcogenides (TMDCs), black phosphorus, etc), have recently been recognized as potential solutions for the aforementioned shortcomings of $3 \mathrm{D}$ noble metals. $\frac{430}{3}$ This view is supported by theoretical calculations which predict that graphene is able to sustain long-lived plasmonic excitations, optoelectronic properties. $\frac{3134}{134}$ At the same time, the electronic density of its charge-carriers can be dynamically tuned by means of electrostatic gating. $.35+37$ The latter provides active control over the plasmon resonance (which is proportional to $\sqrt{E_{F}}$, where $E_{F}$ denotes the Fermi energy of graphene), and therefore may be conveniently varied on-demand. Yet, while the tunability issue was experimentally shown to be lifted by using graphene as a plasmonic 


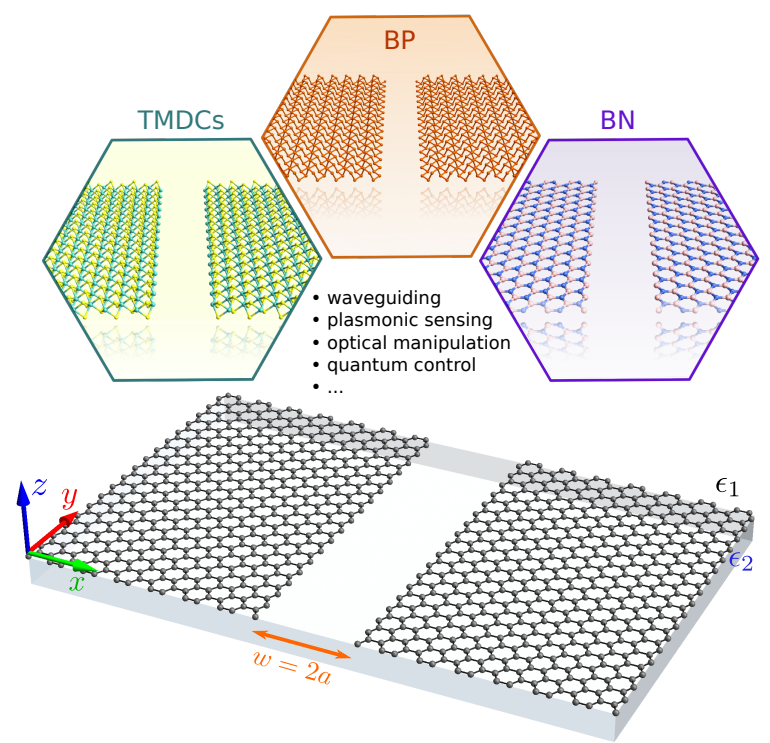

Figure 1: Illustration of the proposed 2D nano-slit using different 2D crystals, including doped graphene, transition metal dichalcogenides (TMDCs) and black phosphorus (BP), and also hexagonal boron nitride $(\mathrm{BN})$. While the latter does not support plasmon-polaritons, it can sustain phonon-polaritons, and thus we include it here since their treatment is analogous. Although only monolayers are portrayed, our framework is also applicable to their few-layer counterparts as long as a $2 \mathrm{D}$ conductivity can be attributed to them.

medium,,$\frac{35+37}{37}$ so far the realization of graphene plasmons ${ }^{31} 33$ with long life-times remains

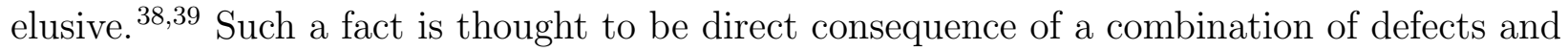
disorder introduced during the growth and/or nanolithography stages, resulting in samples with hindered crystallinity and thereby lower mobilities. Nevertheless, there are reasons to stay optimistic about the promised low-loss graphene plasmonics. Nanofabrication techniques continue to improve, and recently highly confined graphene plasmons with reduced damping were experimentally realized using graphene encapsulated in hexagonal boron nitride (hBN). 40

Graphene plasmons have been observed in various configurations including graphene ribbons, disks or anti-dot arrays. $\sqrt[35+3741-45]{-4 n}$ addition, quasi-1D plasmons sustained at the edge of a semi-infinite graphene layer have been theoretically studied in Refs. 46, 47, based on previous works by Fetter on 2D electron liquids.

In this work, we investigate plasmonic excitations arising in a nano-slit produced in a 2D material capable of sustaining plasmons, such as doped graphene, 2D TMDCs, or phosphorene 
(a black phosphorus monolayer); a scheme of the proposed structure(s) is depicted in Figure 1. The system can be fabricated by stripping a nanoribbon out of an otherwise homogeneous 2D layer, and therefore it is well within reach of current nanolithography techniques. As a more spectacular approach, we here mention the possibility for nanoparticle-assisted writing of nano-slits in graphene. ${ }^{50}$ Finally, ultra-small nanowidths can be achieved by etching from a grain boundary. ${ }^{51}$ We further note that, in the spirit of Babinet's principle,, 5254 our system can be regarded as the complementarity structure to that of a $2 \mathrm{D}$ nanoribbon. For small nano-slit gaps, the Coulomb interaction between edge plasmons in opposite 2D half-sheets gives rise to new hybrid plasmon modes of the compound system. Here, we demonstrate that the coupled plasmon resonances that emerge in such a structure split the dispersion relation of the bare edge plasmon into two new branches: one with lower energy and another with higher energy, akin to a bonding and an antibonding mode in molecular orbital theory. These findings are in line with plasmon hybridization models in metallic nanostructures, introduced in the $1960 \mathrm{~s}^{55}$ and further developed by Nordlander and co-workers. $\frac{56}{56}$ Our results, shown below, rely on a semi-analytical theory based on Green's functions and an orthogonal polynomial expansion $31 / 44 \mid 49157$. This technique allows an accurate and reliable determination of the plasmonic spectrum, as well as the spatial distribution of the potential and corresponding electric field of the modes. ${ }^{31 / 44}$ Naturally, this information may then be used to estimate other related physical quantities, ranging from optical forces to the photonic density of states. Our treatment is comprehensive, encompassing both isotropic and anisotropic atomically thin layers. Furthermore, we have also used commercial-grade full-wave numerical simulations (Lumerical and COMSOL) $\lcm{5859}$ to benchmark our semi-analytical results, to which we have observed an excellent agreement. A particularly attractive feature of our quasi-analytic model is that it entails a universal description of the plasmonic properties of nano-slits made in generic $2 \mathrm{D}$ crystals. This is because, as we detail below, the characteristics of the plasmon resonances depend uniquely on the system's geometry. Consequently, a single calculation is enough to uncover the plasmonic properties in 2D slits of all sizes. Such scale-invariance is 
courtesy of the electrostatic limit. $\frac{60161}{16}$ The consideration of the nonretarded regime here is fully justified since it provides an accurate description of plasmons in 2D materials as the corresponding plasmon wavevectors are typically much larger than $k_{0}=\omega / c . \stackrel{31+33}{[n}$ In passing, we remark that our notion of a $2 \mathrm{D}$ material could in principle also be extended to ultra-thin metallic films (which support a low-frequency plasmon) $\underline{62,64}$

\section{Results and Discussion}

Theoretical Background. We consider an individual nano-slit carved out of an arbitrary 2D crystal, as sketched in Figure 1. We start by giving a brief account of the employed semi-analytic theory. The mathematical details are left as Supporting Information, where they are thoroughly dissected in an all-encompassing fashion. In what follows, we work in the quasistatic limit since the effect of retardation is negligible when studying plasmons in atomically thin materials in typical experiments. Therefore, self-sustained plasmonic excitations in the system are governed by Poisson's equation, $\nabla^{2} \Phi(\mathbf{r})=-\varrho(\mathbf{r}) /\left(\epsilon \epsilon_{0}\right)$. The translation invariance along the $y$-direction (cf. Figure 1) enables us to express the electrostatic potential as $\Phi(\mathbf{r})=\phi(x, z) e^{i k_{y} y}$ (and similarly for the charge-density, $\varrho(\mathbf{r})=\rho(x, z) e^{i k_{y} y}$ ), where a time-dependence of the form $e^{-i \omega t}$ is implicit hereafter. Hence, the Poisson's equation now takes the form

$$
\left[\frac{\partial^{2}}{\partial x^{2}}+\frac{\partial^{2}}{\partial z^{2}}-k_{y}^{2}\right] \phi(x, z)=e \frac{n(x)}{\epsilon \epsilon_{0}} \delta(z)
$$

where we have explicitly written the charge-density in terms of a delta function and a surface carrier density, i.e., $\rho(x, z)=-e n(x) \delta(z)$. The latter can then be expressed as a function of the in-plane electrostatic potential (see Supporting Information), which, together with the Green's function for a planar interface renders a self-consistent integro-differential equation

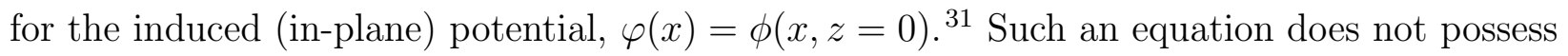
an analytical solution, but one can still make further analytical progress by exploiting: i) the mirror symmetry of the considered nanostructure with respect to the plane bisecting the slit 
(defined by $x=0$ ); and, ii) by expanding the potential in one of the half-sheets using a basis containing Laguerre polynomials. ${ }^{316165}$ In particular, taking $x>0$ without loss of generality, we write $\varphi^{+}(x)=e^{-k_{y}(x-a)} \sum_{n=0}^{\infty} c_{n} L_{n}\left(2 k_{y}[x-a]\right)$-please refer to Supporting Information for a detailed description. These steps are pivotal, and enable us to cast the above stated integro-differential equation into an elementary linear algebra eigenproblem, reading $\underline{66}$

$$
\frac{\epsilon_{1}+\epsilon_{2}}{2 k_{y}} \frac{i \pi \omega \epsilon_{0}}{\sigma_{2 \mathrm{D}}(\omega)} c_{n}=\sum_{m=0}^{\infty} U_{n m} c_{m}
$$

where the set of coefficients $\left\{c_{n}\right\}$ is determined by finding the eigenvectors of the matrix $\mathbf{U}$, whose matrix elements $U_{n m}$ are defined explicitly in the Supporting Information. On the other hand, the eigenvalues of $\mathbf{U}$, hereupon dubbed $\tilde{\lambda}$, uniquely determine the dispersion of the hybrid plasmon excitations guided along the 2D nano-slit, via

$$
\frac{\epsilon_{1}+\epsilon_{2}}{2 k_{y}} \frac{i \pi \omega \epsilon_{0}}{\sigma_{2 \mathrm{D}}(\omega)}=\tilde{\lambda}(\beta)
$$

Clearly, Eq. (3) is completely general irrespective of the particular form of $\sigma_{2 \mathrm{D}}(\omega)$. Thus, provided that one possesses an expression for the 2D conductivity, the plasmonic spectrum directly follows from the condition (3). We stress that, in its present form, Eqs. (2) and (3) assume that the surface conductivity is isotropic. We shall relax this assumption later in the paper, when considering the case of a nano-slit made from an anisotropic 2D material.

Plasmon Dispersion. We now consider - for the sake of illustration and physical insight - a 2D nano-slit made of graphene with a frequency-dependent conductivity given by the Drude model, namely $\sigma_{2 \mathrm{D}}(\omega)=\frac{i e^{2} E_{F}}{\pi \hbar^{2} \omega}$ (assuming negligible losses). Introducing this formula in Eq. (3) yields a closed-form expression for the dispersion of the graphene plasmon resonances in the system

$$
\Omega\left(k_{y}\right)=\sqrt{\frac{2}{\pi} \tilde{\lambda}(\beta)} \Omega_{\mathrm{bulk}}\left(k_{y}\right),
$$

where $\Omega_{\mathrm{bulk}}\left(k_{y}\right)=\sqrt{\frac{4 \alpha E_{F} \hbar c k_{y}}{\epsilon_{1}+\epsilon_{2}}}$ is the nonretarded dispersion relation of plasmons propagating 

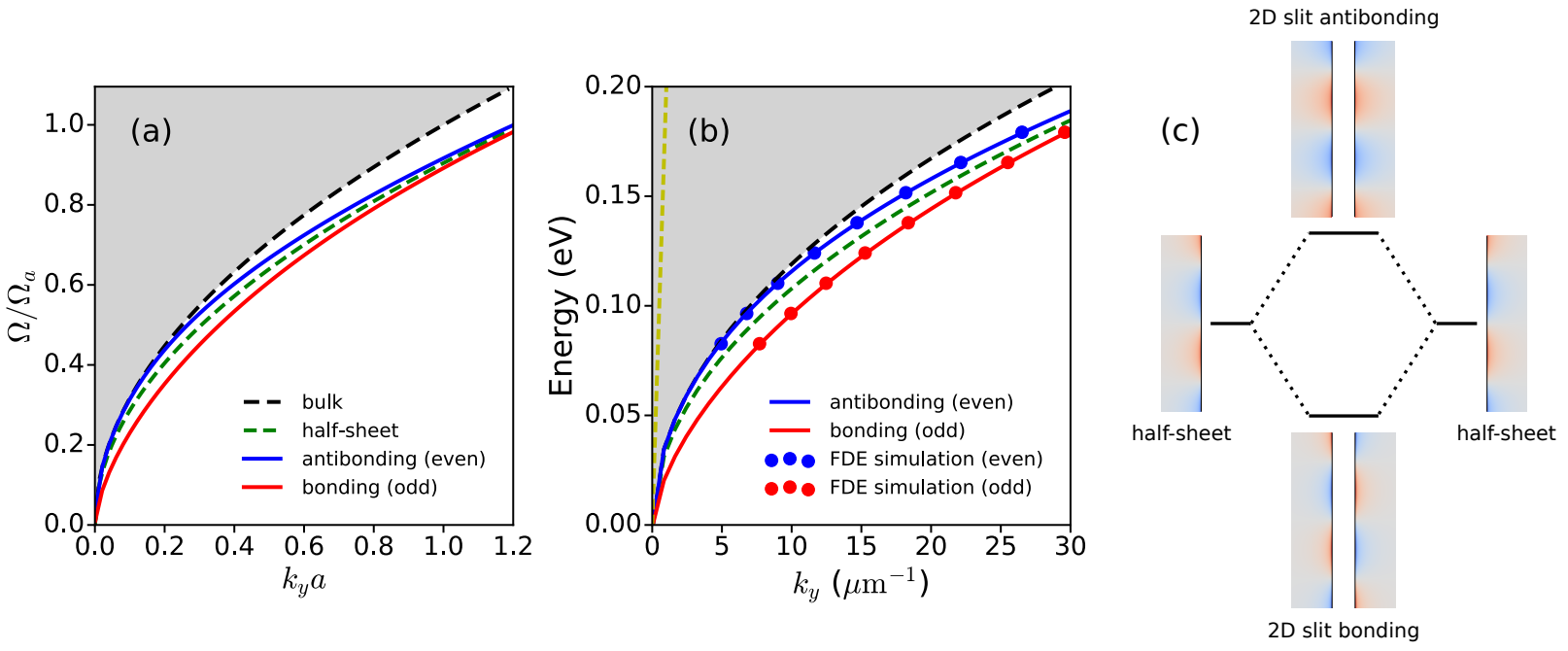

Figure 2: Dispersion relation of hybrid edge plasmons supported by a 2D nano-slit waveguide. The spectrum consist of two plasmonic bands, corresponding to a bonding (odd) and an antibonding (even) mode originating from the interaction between edge plasmons in opposite 2D half-sheets. (a) Spectrum of coupled plasmons in a 2D nano-slit, stemming from Eq. (4), where $\Omega_{a} \equiv \Omega_{\text {bulk }}\left(a^{-1}\right)$. (b) Plasmon dispersion for a representative $w=2 a=50$ nm graphene nano-slit calculated via Eq. (3), where we have employed Kubo's formula at $T=300 \mathrm{~K}$ for the conductivity of graphene (other parameters are $E_{F}=0.5 \mathrm{eV}, \Gamma=3.7 \mathrm{meV}$, and $\epsilon_{1}=\epsilon_{2}=1$ ). The colored circles correspond to data points obtained from full-wave numerical simulations

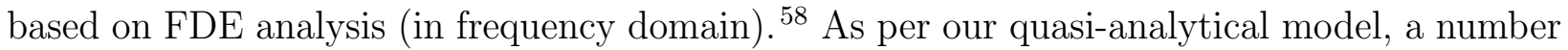
of $N_{\max }=20$ terms were included for the truncation of the matrix $\mathbf{U}$ - see Eq. (2) - , and shown to be sufficient in order to obtain a converged solution. (c) Plasmon hybridization scheme and calculated induced charge-densities ${ }^{67}$ of the plasmon modes, obtained using our model.

with wavevector $k_{y}$ in bulk (i.e., continuous) graphene $e^{31+33}(\alpha \simeq 1 / 137$ denotes the finestructure constant). If the atomically thin layer is instead a $2 \mathrm{D}$ conductor with parabolic dispersion, then expression 44 still holds but now with $\Omega_{\text {bulk }}\left(k_{y}\right)=\sqrt{\frac{4 \pi \alpha(\hbar c)^{3} k_{y}}{\epsilon_{1}+\epsilon_{2}} \frac{n_{e}}{m^{*} c^{2}}}$, where $n_{e}$ and $m^{*}$ are the carrier density and effective mass, respectively 68 . The reader should appreciate that, under its elegant and compact form, Eq. (4) entails a comprehensive description of the effect of the nano-slit's width in the coupling and subsequent hybridization of the modes sustained at opposite edges of the structure. Such information is contained in the eigenvalues $\tilde{\lambda}(\beta)$, which essentially depend on the dimensionless parameter $\beta=k_{y} a$, or, in other words, on the slit width to plasmon wavelength ratio. Therefore, the complete knowledge of the plasmonic spectrum can be fetched by diagonalizing the matrix $\mathbf{U}$ for a 
set of $\beta$-values, and then inserting the determined eigenvalues into Eq. (4). The outcome of such operation is shown in Figure 2. As a guide to the eye, we have also included the spectrum of edge plasmons supported by an individual 2D half-sheet (green dashed line), as well as the plasmonic band of the corresponding bulk 2D plasmon (black dashed line). We stress that the results presented in Figure 2 are valid for an arbitrary (isotropic) 2D crystal (with the appropriate choice of $\Omega_{\mathrm{bulk}}$, as discussed above). Notice that, as we have anticipated, the hybridization between the edge modes of the two half-planes results in a splitting of the unperturbed half-sheet edge plasmons into a pair of new hybrid modes. These arise from antisymmetric and symmetric hybridizations of the bare edge plasmons, giving rise to a bonding and an antibonding branch, lying below and above, respectively, the plasmon band of a single half-sheet-cf. Figure 2, As the name suggests, in the case of the bonding mode the induced charge-density in opposite semi-infinite layers oscillate in anti-phase (odd symmetry), whereas for the antibonding mode such oscillations are in-phase (even symmetry). Naturally, these resonances emerge at different frequencies (for a fixed propagation constant), hence giving rise to the aforementioned energy splitting, as illustrated in Figure 2r.

It is apparent from Figure 2 that, at large wavevectors (in relation to the momentum-scale introduced by $a^{-1}$, i.e., for $k_{y} a \gg 1$ ), both resonances converge asymptotically to that of an edge plasmon in an individual half-plane. In this limit the Coulomb interaction between the neighboring edges falls off rapidly, and therefore the two semi-infinite 2D sheets decouple. Indeed, within this regime, the eigenvalue $\tilde{\lambda}(\beta \rightarrow \infty) \equiv \tilde{\lambda}^{(0)}$ becomes independent of $\beta=k_{y} a$. As a result, the dispersion relation of the modes of the $2 \mathrm{D}$ slit reduces to the plasmon of the unpatterned system multiplied by a proportionality constant, that is, $\Omega\left(k_{y}\right)=\mathrm{C}^{\text {te }} \cdot \Omega_{\mathrm{bulk}}\left(k_{y}\right)$. This behavior can be seen directly by inspecting the (analytical) integral kernel of the matrix elements $U_{n m}$, thanks to the amount of analytical progress performed here-check Supporting Information. This signifies that at large $k_{y} a$ the $2 \mathrm{D}$ nano-slit modes tend to become indistinguishable from that of an single half-sheet. Using our quasi-analytical theory we have obtained $\mathrm{C}^{\mathrm{te}}=\sqrt{2 \tilde{\lambda}^{(0)} / \pi}=0.905$ (see Supporting Information for more details) 
which is in outstanding agreement with the value of $\mathrm{C}^{\text {te }}=0.906$ reported in the literature for edge plasmons supported by a 2D half-plane ${ }^{69}$.

It should also be stressed that Figure 2 a depicts the dispersion of the hybrid plasmon modes of the system in dimensionless units, thus enabling the determination of the plasmonic spectrum in 2D nano-slits of any width, and irrespective of the type of plasmon-supporting 2D material. Necessarily, the exact positions of the resonances depend on the details of the model used for the 2D conductivity, but the general features outlined above should remain qualitatively the same. If the physics of the material cannot be captured by the Drude model, then one simply needs to take a step back and solve Eq. (3) directly, using an expression for the conductivity within the appropriate framework. In that context, Figure $2 \mathrm{~b}$ shows the solution of the condition (3) for a 50nm-wide graphene nano-slit where the graphene has been modeled using Kubo's formula for the conductivity at finite temperature. ${ }^{31}$ In the same figure, we compare the results of our semi-analytical theory against data obtained from numerical full-wave electrodynamic simulations using a commercially available finite-difference

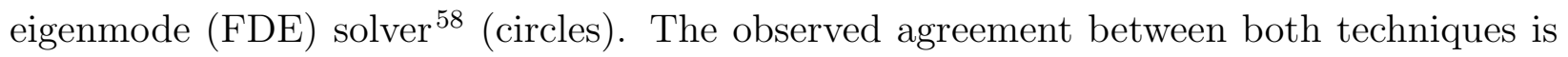
quite remarkable. Such a fact, together with the ability to reproduce the $\beta \rightarrow \infty$ limit, demonstrates the ability of our quasi-analytical model to accurately describe plasmonic excitations in 2D nano-slits. All of this with the added advantage a semi-analytical method provides in portraying a clear and intuitive picture of the physics, without the necessity of relying on often time-consuming numerical simulations.

Potential and Electric-Field Distributions. The solution of the eigenproblem posed by Eq. (2) not only determines the plasmonic spectrum from the eigenvalues $\tilde{\lambda}(\beta)$, but also endow us the scalar potential within the 2D layer directly, by virtue of the eigenvectors of $\mathbf{U}$ (whose entries contain the set of expansion coefficients $\left\{c_{n}\right\}$ ). From here, the electrostatic 


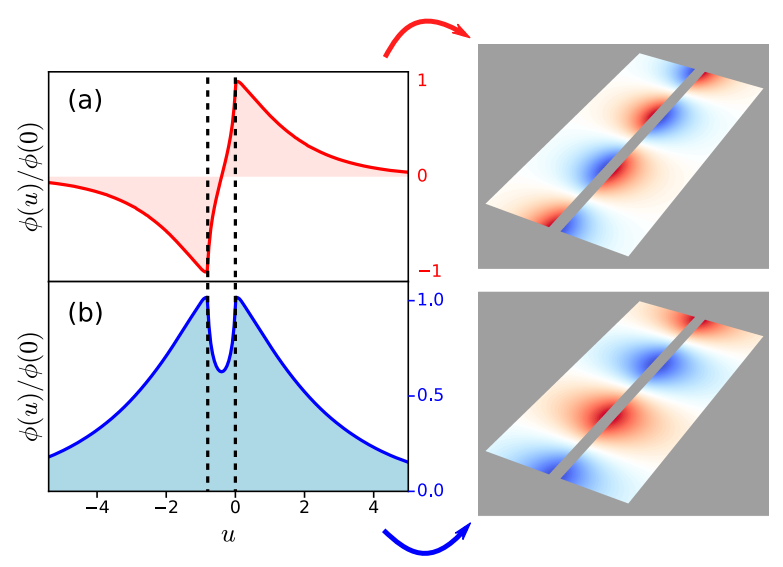

Figure 3: In-plane electrostatic potential along $u=k_{y} x-\beta$, i.e., $\phi(u, \zeta=0)$, akin to the (a) bonding and (b) antibonding hybrid edge plasmons modes, for $\beta=k_{y} a=0.4$. The corresponding panels at the right indicate the electrostatic potential $\Phi\left(u, k_{y} y, 0\right)$ evaluated within the $2 \mathrm{D}$ material. All plots are normalized to the respective maximums.

potential in the entire coordinate space follows from

$$
\begin{aligned}
\phi(u, \zeta) & =-\frac{1}{2 \tilde{\lambda}(\beta)}\left\{\int_{0}^{\infty} d v \mathcal{K}_{\eta}(\beta ; u, v, \zeta)\left[\frac{\partial^{2} \varphi^{+}(v)}{\partial v^{2}}-\varphi^{+}(v)\right]\right. \\
& \left.+\left.\frac{\partial \varphi^{+}(v)}{\partial v}\right|_{v=0} \mathcal{K}_{\eta}(\beta ; u, 0, \zeta)\right\},
\end{aligned}
$$

where we have introduced the dimensionless variables $u=k_{y}(x-a), v=k_{y}\left(x^{\prime}-a\right)$, and $\zeta=k_{y} z$ for numerical convenience and generality, while also defining $\mathcal{K}_{\eta}(\beta ; u, v, \zeta)=$ $K_{0}\left(\sqrt{(u-v)^{2}+\zeta^{2}}\right)+\eta K_{0}\left(\sqrt{(u+v+2 \beta)^{2}+\zeta^{2}}\right)$. Both the bonding and antibonding modes can be obtained in this way upon choosing the parameter $\eta$ appropriately $(\eta=1$ for the antibonding and $\eta=-1$ for the bonding), together with the corresponding $\tilde{\lambda}(\beta)$ and $c_{n}$ 's. Figure 3 illustrates the plasmon-induced in-plane potential, for $\beta=0.4$ (for other values the behavior is qualitatively similar). The corresponding resonant frequencies can be obtained from Figure 2 by reading the intersection of the vertical line $k_{y} a=0.4$ with the dispersion curves. From Figure 3 it is clear that - as already noted above - the bonding (antibonding) mode possesses a potential distribution which is odd (even) with respect to the plane bisecting the nano-slit, with the former exhibiting a nodal line in the middle of the gap.

Finally, the electric field induced by the plasmon oscillations in the system may be readily 
derived from Eq. (5) by taking the gradient of the potential, $\mathbf{E}(\mathbf{r})=-\nabla \Phi(\mathbf{r})$, which gives (componentwise)

$$
\begin{aligned}
& E_{x}(u, \Upsilon, \zeta)=-k_{y} \cos \Upsilon \frac{\partial}{\partial u} \phi(u, \zeta) \\
& E_{y}(u, \Upsilon, \zeta)=k_{y} \sin \Upsilon \phi(u, \zeta) \\
& E_{z}(u, \Upsilon, \zeta)=-k_{y} \cos \Upsilon \frac{\partial}{\partial \zeta} \phi(u, \zeta)
\end{aligned}
$$

where $\Upsilon=k_{y} y$. In possession of the previous expressions, the electric field originating from each of the plasmon resonances can be fully determined at any given point in space. The spatial distributions of all three components of the electric field in the $x z$ - $(u \zeta)$-plane are depicted in Figure 4 (see the caption for details). This figure summarizes the main features of each of the hybrid edge plasmon modes in 2D nano-slits. The field components corresponding to the bonding mode are shown in the uppermost panels, while the ones corresponding to the antibonding mode are pictured in the lower part of the figure. In the main panels we have also superimposed the vector fields, represented by the arrows, associated with each plasmonic excitation. Naturally, the symmetries entailed in the electrostatic potential also emerge here. Interestingly, notice the similarity of the bonding eigenmode with that of an electric dipole oriented across the gap formed by the nano-slit. In contrast, the other resultant mode hybridization resembles two electric monopoles separated by a distance equal to the width of the gap separating the two half-sheets. ${ }^{70}$ Finally, we note that in an experimental setting, a particular plasmonic resonance may appear as "bright" or "dark" under plane-wave illumination depending on the polarization of the incident field. Such criteria could be used, for instance, to select a single mode a priori.

Complementary 2D Structures: Slit and Ribbon. The 2D nano-slit geometry considered here may also be perceived as the inverse (or complementary) structure to a $2 \mathrm{D}$ nanoribbon. Therefore, it is instructive to compare the character of the guided plasmon modes supported by these complementary 2D nanostructures (both assumed to be infinitely 

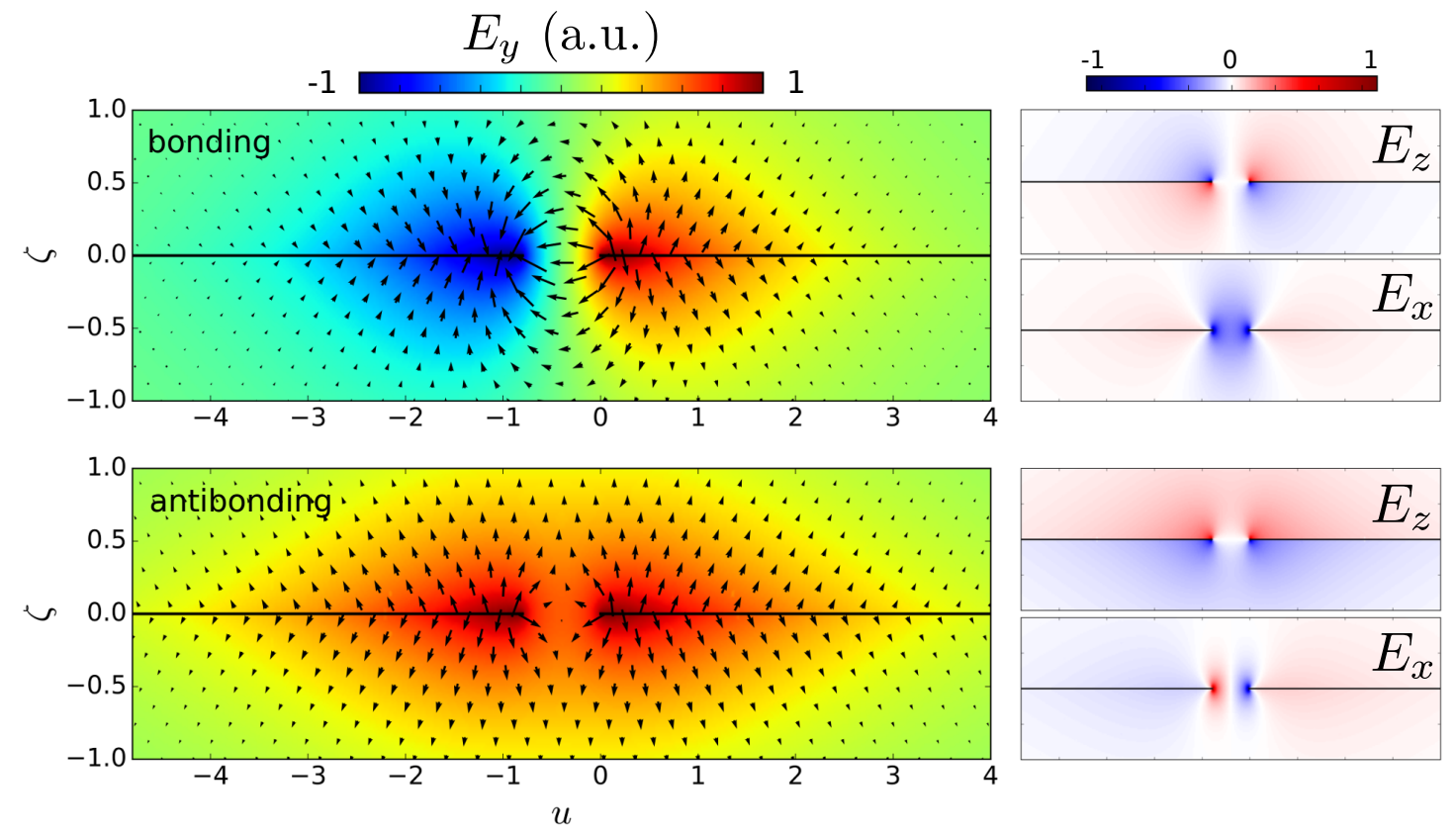

Figure 4: Density plots of the electric field distributions corresponding to the two hybrid edge plasmon modes of a $2 \mathrm{D}$ nano-slit. These are calculated using the quasi-analytic theory described in the text-see Eqs. (5) and (6). We take $\beta=k_{y} a=0.4$, and in the plots the cross-section of the slit is shown. The black solid line indicates the $2 \mathrm{D}$ plasmonic material. The three uppermost panels display the electric field akin to the bonding mode, whereas the ones at the bottom depict the same quantities for the antibonding mode. The vectorial plots superimposed onto the two main panels illustrate the full 2D vector field, $\mathbf{E}(u, \zeta)=E_{x}(u, \zeta) \mathbf{u}_{x}+E_{z}(u, \zeta) \mathbf{u}_{z}$, while the background, in rainbow colors, shows the $y$ component of the electric field (the component parallel to the edges of the nano-slit). The length of the arrows is proportional to the norm of the electric-field vector at that point, $|\mathbf{E}(u, \zeta)|$, in logarithmic scale. Each one of the individual panels are normalized to their own maximum values, and the region depicted in the smaller panels has the same dimensions as the main plots.

long, for the sake of simplicity).

The ribbon system has been object of intensive study, specially inasmuch as graphene is concerned, where it has been shown that graphene ribbons sustain a set of discrete plasmon resonances. $\frac{12[31|35| 36|60| 71 / 72]}{}$ Each of such plasmon excitations are propagating along the ribbon direction, while resembling linear monopoles, dipoles, and higher-order multipoles in the transverse direction. These arise from the confinement of the plasmon wavenumber across the ribbon width, thus mimicking standing waves confined in a one-dimensional box. This contrasts with what we have found for the $2 \mathrm{D}$ nano-slit, and it simply reflects the absence of 
a finite size in the latter case (since the nano-slit is composed by two semi-infinite planes).

Figure 5 shows the dispersion of the hybrid edge plasmons of a 2D slit together with the plasmonic spectrum of a ribbon. We have plotted the dispersion relations in dimensionless units to facilitate the direct comparison between ribbons and slits of the same, but arbitrary, width $w=2 a$. In what follows, we shall focus on the two lowest energy ribbon plasmons, which are the ones that admit a degree of similitude with respect to the modes of a slit. Indeed, analogous to the bonding and antibonding plasmon modes in a 2D slit, the monopole-

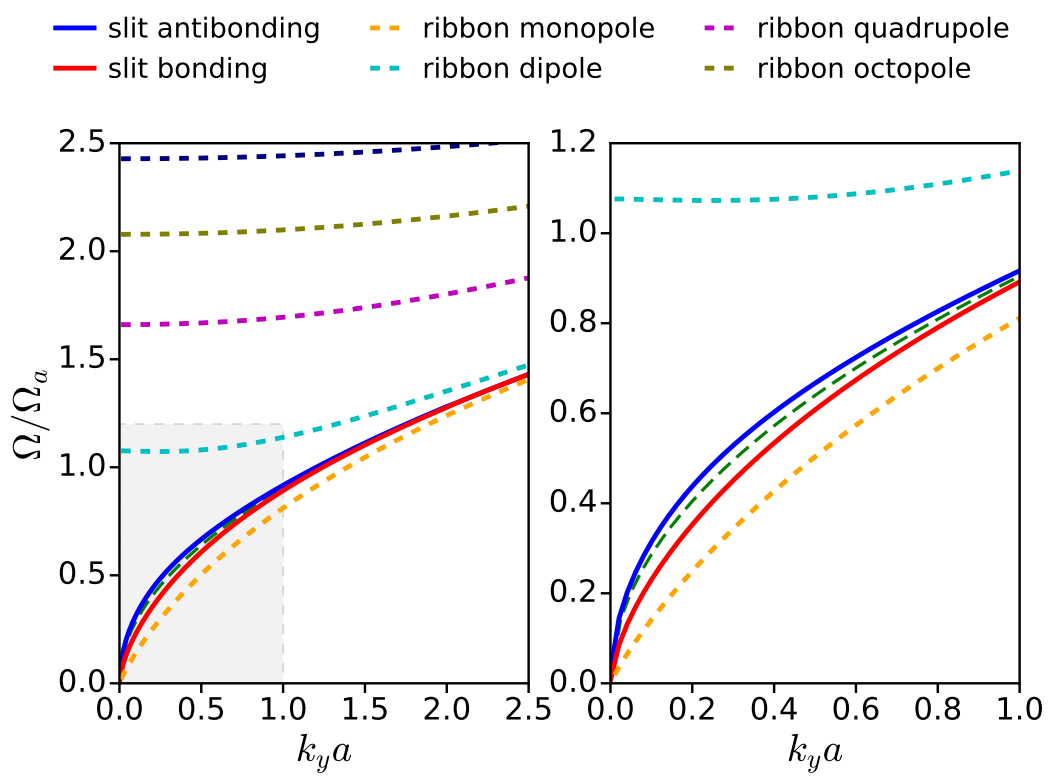

Figure 5: Plots of the dispersion relation of the hybrid edge modes in 2D nano-slits (solid lines) together with the plasmonic spectrum of a nanoribbon (dashed lines). The latter is computed using a similar technique, described elsewhere ${ }^{31}$ The panel at the right is a zoomed version of the shaded area in the left panel. The thin green dashed line represents the edge plasmon in a single half-sheet, and serves as an eye-guide.

and dipole-like ribbon resonances can also be understood as symmetric and antisymmetric hybridizations between the individual half-sheet plasmons living in opposite edges. This interpretation is further substantiated by the fact that both the dispersion of the slit hybrid modes and that of the aforementioned ribbon resonances approach the dispersion curve of the half-sheet edge plasmon at large $k_{y} a$-see Figure 5. In that limit, the plasmons in antipodal edges of the slit/ribbon effectively decouple and become indistinguishable from the edge 
plasmon of a single semi-infinite plane.

At this point, it is worth mentioning the differences and similarities between the $2 \mathrm{D}$ nano-slit and nanoribbon with their equivalent 3D versions, that is, the metal-insulator-metal (MIM) and the insulator-metal-insulator (IMI) structures, respectively. Simply put, we note that the former can be regarded as the lower dimensional form of the latter. In particular, like the $2 \mathrm{D}$ versions discussed here, the MIM and IMI waveguides also accommodate symmetric and antisymmetric hybridizations of the two surface plasmons of the individual insulator-metal interfaces. In addition, although perhaps not surprisingly, both the $2 \mathrm{D}$ slit and the MIM waveguide exhibit a low-frequency plasmon in which the induced charge-density oscillates in anti-phase, whereas for the high-frequency mode they oscillate in-phase; and vice-versa in the ribbon and IMI configurations. However, despite such lookalike features - save for the ones related to the distinct dimensionalities - there are some important differences. First, contrary to the ribbon, the IMI waveguide does not support standing wave like resonances within the thin-film. This can be attributed to the very effective screening within the 3D metal, as opposed to the reduced screening of a 2D ribbon (nevertheless, as soon as the IMI waveguide is patterned into a strip of a thin (but still 3D) film, such standing wave excitations promptly emerge). Lastly, the most interesting distinction between the 2D nano-slit/MIM and their corresponding inverse structures is in what the breaking of complementarity is concerned. It is well-known that, in traditional IMI/MIM waveguides, the dispersion of their plasmon modes is identical in the nonretarded limit. $\frac{64}{6}$ This property is direct consequence of the Babinet's principle of complementarity structures. The complementarity of IMI/MIM waveguides is immediately broken upon inclusion of retardation effects ${ }^{64}$ see also Supporting Information. Incidentally, notice that the situation is remarkably different in the $2 \mathrm{D}$ case. Here, both the 2D slit and ribbon plasmon modes are essentially electrostatic in nature (i.e., retardation effects are negligible), but complementarity seems to be broken nonetheless - cf. Figure 5. This feature has no parallel in the corresponding 3D versions. Strictly speaking, Babinet's principle is only exactly valid for perfect conductors. ${ }^{52}$ Thus, the breaking of complementarity 
may be possibly attributed to the significant extension of the induced electric field within the 2D material, which is comparatively larger than for a 3D metal owing to the skin effect (so that the latter is closer to a perfect conductor).

The apparent breakage of complementarity in mutually inverse $2 \mathrm{D}$ nanostructures is not only interesting from a fundamental viewpoint, but it may also be important in practice when building complex plasmonic structures with mixed solid-inverse geometries, say, for instance, a vertical stack of 2D nano-slit together with a nanoribbon. To the best of our knowledge, such in-depth studies in 2D plasmonic structures are still lacking in the literature (while, e.g., plasmons in graphene nanoholes and nanodisks have been previously studied $\frac{37 / 42}{\text {, }}$ the breaking of complementarity was not investigated). We believe this to be an important topic, certainly deserving future investigations.

Nano-slits of Anisotropic 2D Crystals. In our discussion so far, we have implicitly assumed that the conductivity of the contemplated 2D material is isotropic. This is indeed the case for graphene and group-VI TMDCs such as $\mathrm{MoS}_{2}, \mathrm{WS}_{2}$, or $\mathrm{MoSe}_{2}$. Nevertheless, that remains but a particular case of a broader picture. Notably, there has been a growing interest in the plasmonics of anisotropic 2D materials, either as a platform to enhance and tune their inherent optical birefringence $\sqrt{73} \frac{75}{75}$ or in the context of hyperbolic nanophotonics. ${ }^{76 / 77}$ Examples

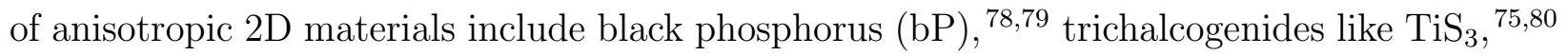
and group-VII TMDCs (for instance, $\mathrm{ReS}_{2}$ ). 181 Among these, few-layer black phosphorus and its monolayer version-phosphorene-have been the subject of remarkable attention from the nanophotonics community, owing both to its high carrier mobility and attractive optical properties. $\frac{79}{}$ For this reason, we will dedicate the next lines to the study of nano-slits made from anisotropic 2D crystals, subsequently focusing on the case of phosphorene.

The key aspect differentiating this case from the isotropic scenario considered above, lies in the fact that the surface conductivity of the atomically thin material is now a tensor. Conveniently, one can still profit from the work performed earlier in the isotropic setting by implementing a few basic modifications in order to contemplate the medium's anisotropy. We 
shall refrain ourselves from enumerating the mathematical details here, but they are provided in the Supporting Information. The corollary of such procedure is an eigenvalue problem resembling the one posed by Eq. (2), where now the matrix $\mathbf{U}$ is augmented by the addition of another matrix accounting for the anisotropy of the system. As before, the dispersion of the plasmon eigenmodes of the anisotropic 2D nano-slit are determined by the eigenvalues of the total matrix. Denoting these by $\xi$, we find that the spectrum of the guided modes in a doped phosphorene nano-slit follows

$$
\Omega_{\mathrm{bP}}\left(k_{y}\right)=\sqrt{\frac{2 \xi(\beta)}{\pi}} \Omega_{2 \mathrm{DEG}}\left(k_{y}\right)
$$

where $\Omega_{2 \mathrm{DEG}}\left(k_{y}\right)$ refers to the dispersion relation of plasmons in a homogeneous and isotropic 2DEG. All the details of the anisotropy and specificities of the 2D crystal are therefore contained in the "anisotropic eigenvalues", $\xi$. Indeed, the same formalism used for phosphorene can also be applied to other anisotropic materials, or even otherwise isotropic materials under the application of uniaxial strain, which effectively breaks the isotropy.

The low-energy bandstructure of monolayer bP can be approximated by that of an ordinary parabolic 2D semiconductor, whose conductivity can be constructed in terms of the carrier effective masses along the high-symmetry directions (zigzag and armchair). ${ }^{76|78| 79}$ Figure 6 shows the dispersion relation of anisotropic plasmons supported by a phosphorene nano-slit doped with electrons. The figure depicts two distinct situations: one where the edges of the slit run along the zigzag direction (left panel), and another in which the nano-slit is parallel to the armchair direction (right panel) - see also figure's insets. From Figure 6, it is clear that although the overall features already seen in the isotropic case remain-namely the existence of a bonding and an antibonding mode, respectively below and above the dispersion of the half-sheet plasmon - the behavior of the eigenmode spectra is strikingly different depending on the orientation of the $2 \mathrm{D}$ nano-slit with respect to the phosphorene's crystal axes. The dramatic contrast between the plasmon dispersion and hybridization in the two cases depicted 


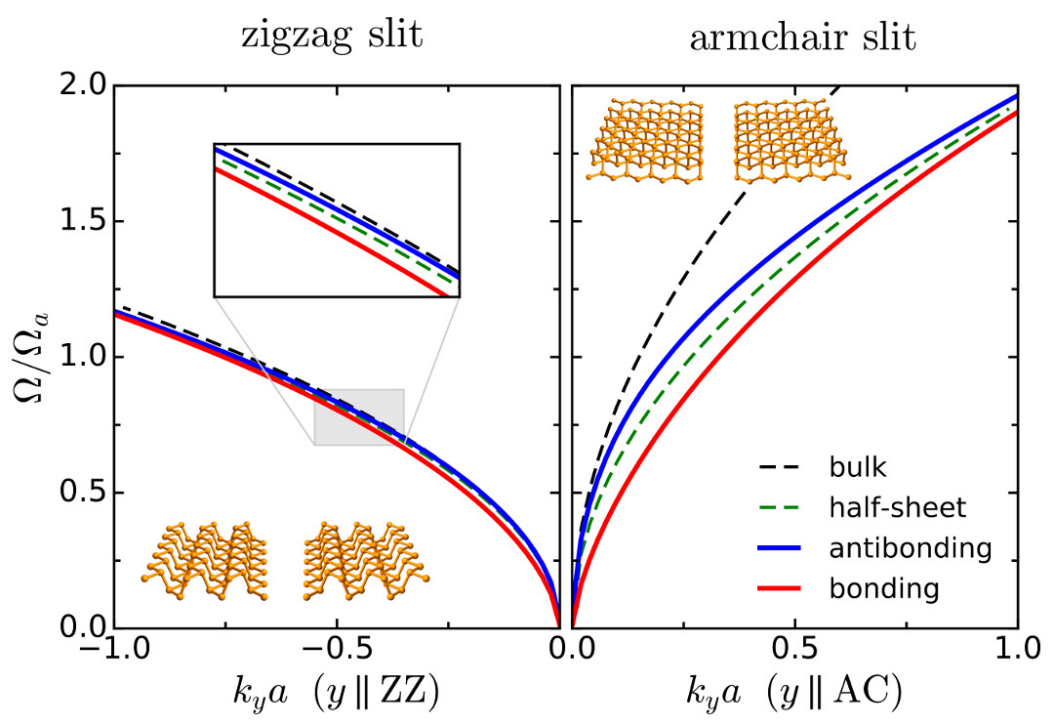

Figure 6: Plasmon eigenmode spectrum of the hybrid modes in a electron doped phosphorene nano-slit. Both the case of a nano-slit patterned along the zigzag and the armchair directions is shown. We have assigned negative values of $k_{y}$ to represent the hybrid plasmonic modes sustained at a zigzag slit, and positive values of $k_{y}$ to identify the eigenmodes of an armchair nano-slit. In our calculations, we take the following parameters for the (anisotropic) electron effective masses: $m_{\mathrm{ZZ}}=0.7 m_{0}$ and $m_{\mathrm{AC}}=0.15 m_{0}, \frac{78779}{{ }^{17}}$ for the electronic bands along the zigzag and armchair directions, respectively. Additionally, here $\Omega_{a}=\Omega_{2 \mathrm{DEG}}\left(k_{y}=a^{-1}\right.$ ) (see Supporting Information).

in Figure 6 reflects the strong anisotropy of black phosphorus (which in turn stems from its puckered honeycomb lattice). Hence, while the dispersion curves associated with the hybrid modes of a zigzag slit are barely indistinguishable from each other (and also from the half-sheet and bulk phosphorene plasmon) in the scale of Figure 6, the splitting between the bonding and antibonding modes of an armchair phosphorene nano-slit is substantial. This arises because, for instance in the latter case, the charge carrier effective mass is substantially lighter along the slit's edges (armchair) and it is heavier in the direction perpendicular to it. Naturally, the situation is reversed in the case of a zigzag nano-slit.

As before, our quasi-analytic results for the anisotropic phosphorene nano-slit, embodied in Figure 6, were benchmarked and subsequently validated by rigorous electrodynamic simulations based on the finite-element method (a comparison is shown in the Supporting Information) $\cdot \underline{59}$ 
Therefore, in this manner, we have demonstrated that our semi-analytical model is extensible to nano-slits made from anisotropic 2D materials as well. Such a fact further enhances the applicability and versatility of our method to describe plasmonic excitations in a wide variety of $2 \mathrm{D}$ plasmonic materials, both with and without anisotropy. We therefore anticipate that our work may contribute to the development of anisotropic 2D plasmonics, since even the nanostructuring of challenging materials, such as phosphorene, is now well within reach of current experimental capabilities. $\underline{82}$

\section{Conclusions and Outlook}

In conclusion, we have conducted a comprehensive theoretical description of the plasmonmediated interaction and subsequent hybridization of plasmons in 2D nano-slits. Using a semi-analytical model, we have shown the emergence of two distinct waveguide-like eigenmodes of the compound system. These consist in a bonding and an antibonding plasmon resonance originating from the cross-talk between the two edge plasmons sustained at opposite margins of the slit. We fully characterize the dispersion relation of such modes, as well as the ensuing potential and electric field distributions. In order to gauge the accurateness of our technique, we also performed full-wave numerical simulations, which remarkably corroborated the results of the former. We note, however, that our semi-analytical framework paints a clearer and intuitive picture of the underlying physics. Furthermore, it also possesses the advantage of being universal, in the sense that - with a single calculation - it renders the plasmon dispersion for 2D slits of any width, and made from an arbitrary 2D material. Going beyond the assumption of a nano-slit constructed from an isotropic 2D medium, the case of a black phosphorus (a strongly anisotropic 2D crystal) nano-slit was also investigated.

Moreover, the slit considered herein can be recognized as the inverse geometry of a ribbon. Therefore, we mutually compared our findings for the slit with the ones for the ribbon case, in the light of Babinet's principle of complementarity. Restricting our considerations to the 
plasmon dispersions, we have observed that Babinet's principle is only strictly satisfied in the asymptotic limit $k_{y} a \rightarrow \infty$. In opposition, in 3D IMI/MIM waveguides, complementarity is maintained within the nonretarded limit. This interesting subject remains largely unexplored in two dimensions, and further research would thus be valuable. Additionally, the combination of complementarity geometries could greatly augment the zoo of 2D plasmonic nanostructures.

In passing, let us underline that the theory described here is within a stone's throw to be exercised in many applications, for instance, to control the decay of quantum emitters (via Purcell enhancement) or for quantum information processing. ${ }^{983}$ The high degree of localization of the electric field near the nano-slit's edges also makes this 2D structure extremely well-suited for plasmonic sensing and SERS. Other applications include the optical manipulation and trapping of nanoparticles in the vicinity of the $2 \mathrm{D}$ nano-slit, due to the large gradients in the electric field intensity. Within the dipole approximation, and neglecting the scattering force, the near-field optical force exerted on the said particle is proportional to $\nabla|\mathbf{E}|^{2}$ (and to the real-part of the particle's polarizability). ${ }^{15}$ Hence, it is immediately apparent from Figure 4 that a dielectric particle will be subjected to significant near-field forces in the neighborhood of the 2D nano-slit (in particular, the trapping potential is deepest at electric field intensity maximums).

Summing up, we have demonstrated that 2D nano-slits are attractive candidates to exploit light-matter interactions at the nanometer scale using the continuously growing family of 2D materials, ranging from deep subwavelength waveguiding and sensing to quantum optical control.

In closing, we emphasize that although here we have focused mostly on plasmon-polaritons, our theory can also be readily used to describe other polaritonic excitations in atomically thin crystals, such as phonon-polaritons in thin hBN slabs, or exciton-polaritons in TMDCs. $\underline{617}$ We believe that our investigation contributes with a new building block - a one-atom-thick nano-slit - to the 2D toolkit of hybrid plasmon resonances, thereby expanding our freedom and capabilities to design new tunable plasmonic systems based on flatland plasmonics. 


\section{Acknowledgement}

P.A.D.G., S.X., and N.A.M. acknowledge the Danish National Research Foundation (Project DNRF103), through the Center for Nanostructured Graphene. N.A.M. is a VILLUM Investigator supported by the VILLUM FONDEN (grant No. 16498). N.M.R.P. acknowledges financial support from the European Commission (696656), and the Fundação Portuguesa para a Ciência e a Tecnologia (UID/FIS/04650/2013).

\section{Supporting Information Available}

The following files are available free of charge. Supporting Information Available: In the Supporting Information we describe, in detail, the derivation of the semi-analytical model outlined here. Both cases, that of a 2D nano-slit made out of an isotropic 2D material and out of an anisotropic one, are addressed. We further comment on the effect of losses on the plasmon propagation and life-time. Finally, we briefly discuss the plasmon dispersion in complementarity $3 \mathrm{D}$ and 2D structures.

\section{References}

(1) Atwater, H. A. The Promise of Plasmonics. Sci. Am. 2007, 296, 56-62.

(2) Gramotnev, D. K.; Bozhevolnyi, S. I. Plasmonics beyond the diffraction limit. Nat. Photon. 2010, 4, 83-91.

(3) Barnes, W. L.; Dereux, A.; Ebbesen, T. W. Surface plasmon subwavelength optics. Nature 2003, 424, 824-830.

(4) Fernández-Domínguez, A. I.; García-Vidal, F. J.; Martín-Moreno, L. Unrelenting plasmons. Nat. Photon. 2017, 11, 8-10. 
(5) Xia, F.; Wang, H.; Xiao, D.; Dubey, M.; Ramasubramaniam, A. Two-dimensional material nanophotonics. Nat. Photon. 2014, 8, 899-907.

(6) Basov, D. N.; Fogler, M. M.; García de Abajo, F. J. Polaritons in van der Waals materials. Science 2016, 354 .

(7) Low, T.; Chaves, A.; Caldwell, J. D.; Kumar, A.; Fang, N. X.; Avouris, P.; Heinz, T. F.; Guinea, F.; Martín-Moreno, L.; Koppens, F. Polaritons in layered two-dimensional materials. Nat. Mater. 2016,

(8) Tame, M. S.; McEnery, K. R.; Ozdemir, S. K.; Lee, J.; Maier, S. A.; Kim, M. S. Quantum plasmonics. Nat. Phys. 2013, 9, 329-340.

(9) Bozhevolnyi, S. I.; Mortensen, N. A. Plasmonics for emerging quantum technologies. Nanophotonics 2017, 6, 1185-1188.

(10) Brolo, A. G. Plasmonics for future biosensors. Nat. Photon. 2012, 6, 709-713.

(11) Aćimović, S. S.; Ortega, M. A.; Sanz, V.; Berthelot, J.; Garcia-Cordero, J. L.; Renger, J.; Maerkl, S. J.; Kreuzer, M. P.; Quidant, R. LSPR Chip for Parallel, Rapid, and Sensitive Detection of Cancer Markers in Serum. Nano Lett. 2014, 14, 2636-2641.

(12) Rodrigo, D.; Limaj, O.; DavideJanner,; Etezadi, D.; García de Abajo, F. J.; Pruneri, V.; Altug, H. Mid-infrared plasmonic biosensing with graphene. Science 2015, 349, 165-168.

(13) Haes, A. J.; Haynes, C. L.; McFarland, A. D.; Schatz, G. C.; Van Duyne, R. P.; Zou, S. Plasmonic Materials for Surface-Enhanced Sensing and Spectroscopy. MRS Bulletin 2005, 30, 368-375.

(14) Raziman, T. V.; Wolke, R. J.; Martin, O. J. F. Optical forces in nanoplasmonic systems: how do they work, what can they be useful for? Faraday Discuss. 2015, 178, 421-434.

(15) Novotny, L.; Hecht, B. Principles of Nano-Optics, 2nd ed.; Cambridge University Press, 2012. 
(16) Kauranen, M.; Zayats, A. V. Nonlinear plasmonics. Nat. Photon. 2012, 6, 737-748.

(17) Metzger, B.; Hentschel, M.; Giessen, H. Ultrafast Nonlinear Plasmonic Spectroscopy: From Dipole Nanoantennas to Complex Hybrid Plasmonic Structures. ACS Photonics 2016, 3, 1336-1350.

(18) Anger, P.; Bharadwaj, P.; Novotny, L. Enhancement and Quenching of Single-Molecule Fluorescence. Phys. Rev. Lett. 2006, 96, 113002.

(19) Pors, A.; Bozhevolnyi, S. I. Quantum Emitters near Layered Plasmonic Nanostructures: Decay Rate Contributions. ACS Photonics 2015, 2, 228-236.

(20) Smith, C. L. C.; Stenger, N.; Kristensen, A.; Mortensen, N. A.; Bozhevolnyi, S. I. Gap and channeled plasmons in tapered grooves: a review. Nanoscale 2015, 7, 9355-9386.

(21) Raza et al., S. Extremely confined gap surface-plasmon modes excited by electrons. Nat. Commun. 2014, 5, 4125.

(22) Zohar, N.; Chuntonov, L.; Haran, G. The simplest plasmonic molecules: Metal nanoparticle dimers and trimers. J. Photochem. Photobiol. C 2014, 21, 26-39.

(23) Dodson, S.; Haggui, M.; Bachelot, R.; Plain, J.; Li, S.; Xiong, Q. Optimizing Electromagnetic Hotspots in Plasmonic Bowtie Nanoantennae. J. Phys. Chem. Lett. 2013, 4, 496-501.

(24) Zhu, W.; Esteban, R.; Borisov, A. G.; Baumberg, J. J.; Nordlander, P.; Lezec, H. J.; Aizpurua, J.; Crozier, K. B. Quantum mechanical effects in plasmonic structures with subnanometre gaps. Nat. Commun. 2016, 7, 11495.

(25) Chikkaraddy, R.; Zheng, X.; Benz, F.; Brooks, L. J.; de Nijs, B.; Carnegie, C.; Kleemann, M.-E.; Mertens, J.; Bowman, R. W.; Vandenbosch, G. A. E.; Moshchalkov, V. V.; Baumberg, J. J. How Ultranarrow Gap Symmetries Control Plasmonic Nanocavity 
Modes: From Cubes to Spheres in the Nanoparticle-on-Mirror. ACS Photonics 2017, 4, $469-475$.

(26) Chikkaraddy, R.; de Nijs, B.; Benz, F.; Barrow, S. J.; Scherman, O. A.; Rosta, E.; Demetriadou, A.; Fox, P.; Hess, O.; Baumberg, J. J. Single-molecule strong coupling at room temperature in plasmonic nanocavities. Nature 2016, 535, 127-130.

(27) Benz et al., F. Single-molecule optomechanics in "picocavities". Science 2016, 354 , 726-729.

(28) Sigle, D. O.; Mertens, J.; Herrmann, L. O.; Bowman, R. W.; Ithurria, S.; Dubertret, B.; Shi, Y.; Yang, H. Y.; Tserkezis, C.; Aizpurua, J.; Baumberg, J. J. Monitoring Morphological Changes in 2D Monolayer Semiconductors Using Atom-Thick Plasmonic Nanocavities. ACS Nano 2015, 9, 825-830.

(29) Mertens, J.; Eiden, A. L.; Sigle, D. O.; Huang, F.; Lombardo, A.; Sun, Z.; Sundaram, R. S.; Colli, A.; Tserkezis, C.; Aizpurua, J.; Milana, S.; Ferrari, A. C.; Baumberg, J. J. Controlling Subnanometer Gaps in Plasmonic Dimers Using Graphene. Nano Lett. 2013, 13, 5033-5038.

(30) Koppens, F. H. L.; Chang, D. E.; García de Abajo, F. J. Graphene Plasmonics: A Platform for Strong Light-Matter Interactions. Nano Lett. 2011, 11, 3370-3377.

(31) Gonçalves, P. A. D.; Peres, N. M. R. An Introduction to Graphene Plasmonics, 1st ed.; World Scientific: Singapore, 2016.

(32) García de Abajo, F. J. Graphene Plasmonics: Challenges and Opportunities. ACS Photonics 2014, 1, 135-152.

(33) Xiao, S.; Zhu, X.; Li, B.-H.; Mortensen, N. A. Graphene-plasmon polaritons: From fundamental properties to potential applications. Front. Phys. 2016, 11, 117801. 
(34) Castro Neto, A. H.; Guinea, F.; Peres, N. M. R.; Novoselov, K. S.; Geim, A. K. The electronic properties of graphene. Rev. Mod. Phys. 2009, 81, 109-162.

(35) Ju, L.; Geng, B.; Horng, J.; Girit, C.; Martin, M.; Hao, Z.; Bechtel, H. A.; Liang, X.; Zettl, A.; Shen, Y. R.; Wang, F. Graphene plasmonics for tunable terahertz metamaterials. Nat. Nanotechnol. 2011, 6, 630-634.

(36) Yan, H.; Low, T.; Zhu, W.; Wu, Y.; Freitag, M.; Li, X.; Guinea, F.; Avouris, P.; Xia, F. Damping pathways of mid-infrared plasmons in graphene nanostructures. Nat. Photon. 2013, 7, 394-399.

(37) Fang, Z.; Thongrattanasiri, S.; Schlather, A.; Liu, Z.; Ma, L.; Wang, Y.; Ajayan, P. M.; Nordlander, P.; Halas, N. J.; García de Abajo, F. J. Gated Tunability and Hybridization of Localized Plasmons in Nanostructured Graphene. ACS Nano 2013, 7, 2388-2395.

(38) Tassin, P.; Koschny, T.; Kafesaki, M.; Soukoulis, C. M. A comparison of graphene, superconductors and metals as conductors for metamaterials and plasmonics. Nat. Photon. 2012, 6, 259-264.

(39) Dastmalchi, B.; Tassin, P.; Koschny, T.; Soukoulis, C. M. A New Perspective on Plasmonics: Confinement and Propagation Length of Surface Plasmons for Different Materials and Geometries. Adv. Opt. Mater. 2016, 4, 177-184.

(40) Woessner et al., A. Highly confined low-loss plasmons in graphene-boron nitride heterostructures. Nat. Mater. 2015, 14, 421-425.

(41) Yan, H.; Xia, F.; Li, Z.; Avouris, P. Plasmonics of coupled graphene micro-structures. New J. Phys. 2012, 14, 125001.

(42) Zhu, X.; Wang, W.; Yan, W.; Larsen, M. B.; Bøggild, P.; Pedersen, T. G.; Xiao, S.; Zi, J.; Mortensen, N. A. Plasmon-Phonon Coupling in Large-Area Graphene Dot and Antidot Arrays Fabricated by Nanosphere Lithography. Nano Lett. 2014, 14, 2907-2913. 
(43) Wang, Z.; Li, T.; Almdal, K.; Mortensen, N. A.; Xiao, S.; Ndoni, S. Experimental demonstration of graphene plasmons working close to the near-infrared window. Opt. Lett. 2016, 41, 5345-5348.

(44) Gonçalves, P. A. D.; Dias, E. J. C.; Xiao, S.; Vasilevskiy, M. I.; Mortensen, N. A.; Peres, N. M. R. Graphene Plasmons in Triangular Wedges and Grooves. ACS Photonics 2016, 3, 2176-2183.

(45) Gonçalves, P. A. D.; Bozhevolnyi, S. I.; Mortensen, N. A.; Peres, N. M. R. Universal description of channel plasmons in two-dimensional materials. Optica 2017, 4, 595-600.

(46) Wang, W.; Apell, P.; Kinaret, J. Edge plasmons in graphene nanostructures. Phys. Rev. B 2011, 84, 085423 .

(47) Wang, W.; Kinaret, J. M.; Apell, S. P. Excitation of edge magnetoplasmons in semiinfinite graphene sheets: Temperature effects. Phys. Rev. B 2012, 85, 235444.

(48) Mast, D. B.; Dahm, A. J.; Fetter, A. L. Observation of Bulk and Edge Magnetoplasmons in a Two-Dimensional Electron Fluid. Phys. Rev. Lett. 1985, 54, 1706-1709.

(49) Fetter, A. L. Edge magnetoplasmons in a two-dimensional electron fluid confined to a half-plane. Phys. Rev. B 1986, 33, 3717-3723.

(50) Booth, T. J.; Pizzocchero, F.; Andersen, H.; Hansen, T. W.; Wagner, J. B.; Jinschek, J. R.; Dunin-Borkowski, R. E.; Hansen, O.; Bøggild, P. Discrete Dynamics of Nanoparticle Channelling in Suspended Graphene. Nano Lett. 2011, 11, 2689-2692.

(51) Xie, L.; Liao, M.; Wang, S.; Yu, H.; Du, L.; Tang, J.; Zhao, J.; Zhang, J.; Chen, P.; Lu, X.; Wang, G.; Xie, G.; Yang, R.; Shi, D.; Zhang, G. Graphene-Contacted Ultrashort Channel Monolayer MoS2 Transistors. Advanced Materials 2017, 1702522.

(52) Born, M.; Wolf, E. Principles of Optics, 7th ed.; Cambridge University Press, 1999. 
(53) Zentgraf, T.; Meyrath, T. P.; Seidel, A.; Kaiser, S.; Giessen, H.; Rockstuhl, C.; Lederer, F. Babinet's principle for optical frequency metamaterials and nanoantennas. Phys. Rev. $B$ 2007, 76, 033407.

(54) Ögüt, B.; Vogelgesang, R.; Sigle, W.; Talebi, N.; Koch, C. T.; van Aken, P. A. Hybridized Metal Slit Eigenmodes as an Illustration of Babinet's Principle. ACS Nano 2011, 5, $6701-6706$.

(55) Economou, E. N. Surface Plasmons in Thin Films. Phys. Rev. 1969, 182, 539-554.

(56) Prodan, E.; Radloff, C.; Halas, N. J.; Nordlander, P. A Hybridization Model for the Plasmon Response of Complex Nanostructures. Science 2003, 302, 419-422.

(57) Wang, W.; Christensen, T.; Jauho, A.-P.; Thygesen, K. S.; Wubs, M.; Mortensen, N. A. Plasmonic eigenmodes in individual and bow-tie graphene nanotriangles. Sci. Rep. 2015, 5,9535 .

(58) Lumerical Solutions Inc., MODE Solutions. http://www.lumerical.com/tcadproducts/mode/.

(59) COMSOL Multiphysics, www.comsol.com.

(60) Christensen, J.; Manjavacas, A.; Thongrattanasiri, S.; Koppens, F. H. L.; García de Abajo, F. J. Graphene Plasmon Waveguiding and Hybridization in Individual and Paired Nanoribbons. ACS Nano 2012, 6, 431-440.

(61) Jackson, J. D. Classical Electrodynamics, 3rd ed.; Wiley: New York, 1998.

(62) Lee, S.; Kim, S. Long-range channel plasmon polaritons in thin metal film V-grooves. Opt. Express 2011, 19, 9836-9847.

(63) Chen, X.; Lindquist, N. C.; Klemme, D. J.; Nagpal, P.; Norris, D. J.; Oh, S.-H. SplitWedge Antennas with Sub-5 nm Gaps for Plasmonic Nanofocusing. Nano Lett. 2016, $16,7849-7856$. 
(64) Raza, S.; Christensen, T.; Wubs, M.; Bozhevolnyi, S. I.; Mortensen, N. A. Nonlocal response in thin-film waveguides: Loss versus nonlocality and breaking of complementarity. Phys. Rev. B 2013, 88, 115401, Incidentally, here the incorporation of nonlocal effects was shown to break the complementarity in IMI/MIM waveguides even in the nonretarded limit. We do not, however, bring this up in the main text since we are treating the 2D material in the local response approximation, and therefore such comparison would be fruitless.

(65) Abramowitz, M.; Stegun, I. A. Handbook of Mathematical Functions: with Formulas, Graphs, and Mathematical Tables; Dover: New York, 1965.

(66) Notice that we have replaced $\epsilon$ by $\left(\epsilon_{1}+\epsilon_{2}\right) / 2$ to correctly account for the generic case of two different dielectric media (as shown in Figure 1). We should stress that this no approximation, but simply a consequence of the electrostatic limit and a planar interface.

(67) In the density plots exhibited in Figure 2c the the slit width has been slightly exaggerated for illustration purposes only. The correct scaling is, however, shown in Figure 3 when plotting the potential.

(68) Recall that the Drude conductivity of a 2DEG is given by $\sigma_{2 \mathrm{D}}(\omega)=\frac{i e^{2}}{\omega} \frac{n_{e}}{m^{*}}$, in the absence of damping.

(69) Volkov, V. A.; Mikhailov, S. A. Edge magnetoplasmons: low-frequency weakly damped excitations in inhomogeneous two-dimensional electron systems. Sov. Phys. JETP 1988, $67,1639-1653$, Here, the authors calculated the constant $\mathrm{C}^{\text {te }}=\eta_{0}^{-1 / 2}$ analytically using the Wiener-Hopf technique, and stems from the root of the integral $\int_{0}^{\pi / 2} d x \ln \left[\eta_{0} / \sin x-\right.$ $1]=0$.

(70) A note of caution is in order here. Although at this point we have used the words 'dipole' and 'monopole', this is actually to be interpreted as a line -in the center of the slit 
and running parallel to its edges - of alternating dipoles or monopoles. For instance, ordinary dipoles possess cylindrical symmetry whereas in the current case the symmetry is with respect to the $z=0$ plane.

(71) Zhu, X.; Yan, W.; Mortensen, N. A.; Xiao, S. Bends and splitters in graphene nanoribbon waveguides. Opt. Express 2013, 21, 3486-3491.

(72) Gonçalves, P. A. D.; Dias, E. J. C.; Bludov, Y. V.; Peres, N. M. R. Modeling the excitation of graphene plasmons in periodic grids of graphene ribbons: An analytical approach. Phys. Rev. B 2016, 94, 195421.

(73) Khaliji, K.; Fallahi, A.; Martin-Moreno, L.; Low, T. Tunable plasmon-enhanced birefringence in ribbon array of anisotropic two-dimensional materials. Phys. Rev. B 2017, 95, 201401.

(74) Yang, H.; Jussila, H.; Autere, A.; Komsa, H.-P.; Ye, G.; Chen, X.; Hasan, T.; Sun, Z. Optical Waveplates Based on Birefringence of Anisotropic Two-Dimensional Layered Materials. ACS Photonics 2017, Article ASAP, DOI: 10.1021/acsphotonics.7b00507.

(75) Silva-Guillén, J. A.; Canadell, E.; Ordejón, P.; Guinea, F.; Roldán, R. Anisotropic features in the electronic structure of the two-dimensional transition metal trichalcogenide TiS 3 : electron doping and plasmons. 2D Materials 2017, 4, 025085.

(76) Nemilentsau, A.; Low, T.; Hanson, G. Anisotropic 2D Materials for Tunable Hyperbolic Plasmonics. Phys. Rev. Lett. 2016, 116, 066804.

(77) Gjerding, M. N.; Petersen, R.; Pedersen, T. G.; Mortensen, N. A.; Thygesen, K. S. Layered van der Waals crystals with hyperbolic light dispersion. Nat. Commun. 2017, 8,320 .

(78) Low, T.; Roldán, R.; Wang, H.; Xia, F.; Avouris, P.; Moreno, L. M.; Guinea, F. Plasmons 
and Screening in Monolayer and Multilayer Black Phosphorus. Phys. Rev. Lett. 2014, $113,106802$.

(79) Carvalho, A.; Wang, M.; Zhu, X.; Rodin, A. S.; Su, H.; Neto, A. H. C. Phosphorene: from theory to applications. Nat. Rev. Mater. 2016, 1, 16061.

(80) Dai, J.; Zeng, X. C. Titanium Trisulfide Monolayer: Theoretical Prediction of a New Direct-Gap Semiconductor with High and Anisotropic Carrier Mobility. Ang. Chem. 2015, 127, 7682-7686.

(81) Rahman, M.; Davey, K.; Qiao, S.-Z. Advent of 2D Rhenium Disulfide (ReS2): Fundamentals to Applications. Adv. Func. Mater. 2017, 27, 1606129, 1606129.

(82) Cupo, A.; Masih Das, P.; Chien, C.-C.; Danda, G.; Kharche, N.; Tristant, D.; Drndić, M.; Meunier, V. Periodic Arrays of Phosphorene Nanopores as Antidot Lattices with Tunable Properties. ACS Nano 2017, 11, 7494-7507.

(83) Chang, D. E.; Sørensen, A. S.; Hemmer, P. R.; Lukin, M. D. Quantum Optics with Surface Plasmons. Phys. Rev. Lett. 2006, 97, 053002.

(84) Marquier, F.; Sauvan, C.; Greffet, J.-J. Revisiting Quantum Optics with Surface Plasmons and Plasmonic Resonators. ACS Photonics 2017, Article ASAP, DOI: 10.1021/acsphotonics.7b00475. 